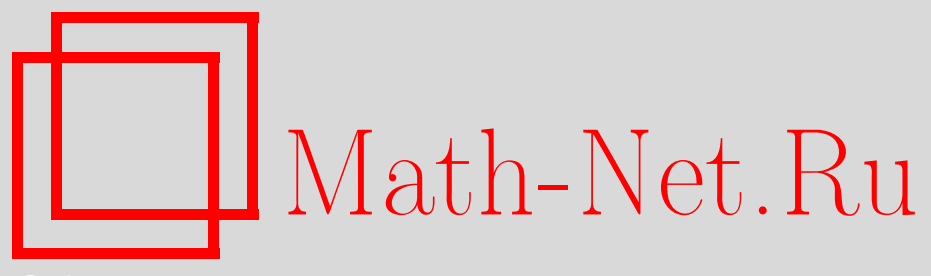

А. А. Илларионов, О статистических свойствах многогранников Клейна трехмерных целочисленных решеток, Матем. сб., 2013, том 204, номер 6, 23-46

DOI: https://doi.org/10.4213/sm7826

Использование Общероссийского математического портала Math-Net.Ru подразумевает, что вы прочитали и согласны с пользовательским соглашением http://www . mathnet.ru/rus/agreement

Параметры загрузки:

IP: 107.22 .136 .117

26 апреля 2023 г., 17:04:20

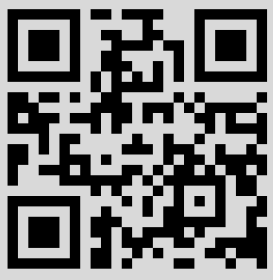




\section{А. А. Илларионов \\ О статистических свойствах многогранников Клейна трехмерных целочисленных решеток}

Получены асимптотические формулы для средних значений количества граней фиксированного типа и вершин полиэдров Клейна трехмерных целочисленных решеток с заданным определителем.

Библиография: 20 названий.

Ключевые слова: решетка, многогранник Клейна, многомерная непрерывная дробь, относительный минимум.

DOI: $10.4213 / \operatorname{sm} 7826$

\section{Обозначения}

$\# S$ - количество элементов конечного множества $S$;

Conv $X$ - выпуклая оболочка множества $X$;

если $x=\left(x_{1}, \ldots, x_{s}\right) \in \mathbb{R}^{s}$, то $|x|=\left(x_{1}^{2}+\cdots+x_{s}^{2}\right)^{1 / 2}$;

$\zeta(s)$ - дзета-функция Римана;

$\mathscr{M}_{s}(X)$ - множество матриц размера $s \times s$ с элементами из $X$, где $X=\mathbb{R}$ или $X=\mathbb{Z}$;

$\mathrm{GL}_{s}(\mathbb{R})$ - множество невырожденных матриц из $\mathscr{M}_{s}(\mathbb{R}) ;$

$\mathrm{GL}_{s}(\mathbb{Z})$ - множество матриц из $\mathscr{M}_{s}(\mathbb{Z})$ с определителем \pm 1 ; если $a^{(1)}, \ldots, a^{(s)} \in \mathbb{R}^{s}$, то

$$
M\left(a^{(1)}, \ldots, a^{(s)}\right)=\left(\begin{array}{ccc}
a_{1}^{(1)} & \ldots & a_{1}^{(s)} \\
\vdots & & \vdots \\
a_{s}^{(1)} & \ldots & a_{s}^{(s)}
\end{array}\right)
$$

- матрица со столбцами $a^{(1)}, \ldots, a^{(s)}$;

если $M \in \mathscr{M}_{s}(\mathbb{R}), X \subset \mathbb{R}^{s}$, то $M \cdot X=\{M \cdot x: x \in X\} \subset \mathbb{R}^{s}$;

запись

$$
f(x) \ll g(x) \quad \text { (либо } f(x)=O(g(x))) \quad \text { при } x \in X
$$

означает, что существует такая постоянная $C>0$, что $|f(x)| \leqslant C \cdot g(x)$ при всех $x \in X$. Если $C$ зависит от параметра $\theta$, то пишем $f(x) \underset{\theta}{\ll} g(x)$ (либо $f(x)=O_{\theta}(g(x))$ ). Запись $f \asymp g$ означает что $f \ll g \ll f$.

Работа выполнена при поддержке Российского фонда фундаментальных исследований (грант № 11-01-00628-а), ДВО РАН (проект № 12-I-B-П19-01) и Программы Президента РФ поддержки ведущих научных школ (грант № НШ-1922.2012.1).

(C) А.А. ИллАрионов, 2013 


\section{Введение}

Полной $s$-мерной решеткой называется множество вида

$$
\Gamma=\left\{k_{1} a^{(1)}+\cdots+k_{s} a^{(s)}: k_{i} \in \mathbb{Z}, i=\overline{1, s}\right\},
$$

где $a^{(i)}, i=\overline{1, s},-$ линейно независимые векторы из $\mathbb{R}^{s}$ (базис $\Gamma$ ). Матрицу $M=M\left(a^{(1)}, \ldots, a^{(s)}\right)$ будем называть базисной (столбцы $M$ образуют базис $\left.\Gamma\right)$. Величина $\operatorname{det} \Gamma=|\operatorname{det} M|$ называется определителем решетки $\Gamma$.

Ненулевой узел $\gamma \in \Gamma$ называется относителъным минимумом s-мерной решетки $\Gamma$, если не существует ненулевого узла $\gamma^{\prime} \in \Gamma$, для которого

$$
\left|\gamma_{i}^{\prime}\right| \leqslant\left|\gamma_{i}\right|, \quad i=\overline{1, s}, \quad|\gamma|<\left|\gamma^{\prime}\right|
$$

Многогранниками (полиэдрами) Клейна решетки Г называются множества вида $\mathscr{K}_{\theta}(\Gamma)$, где $\theta=\left(\theta_{1}, \ldots, \theta_{s}\right), \theta_{i}= \pm 1$, которые определяются как выпуклая оболочка ненулевых узлов $\Gamma$, содержащихся в $s$-гранном угле

$$
\left\{x \in \mathbb{R}^{s}: x_{i} \theta_{i} \geqslant 0, i=\overline{1, s}\right\} .
$$

Будем придерживаться следующих обозначений:

$\mathscr{L}_{s}(X)$ - множество $s$-мерных решеток из $X^{s}$, где $X=\mathbb{R}$ или $X=\mathbb{Z}$;

$\mathscr{L}_{s}(X ; N)$ - множество решеток из $\mathscr{L}_{s}(X)$ с определителем $N$;

$\mathfrak{M}(\Gamma)$ - множество относительных минимумов решетки $\Gamma$;

$\mathscr{V}(\Gamma)$ - множество вершин многогранников Клейна решетки $Г$.

Понятие относительного минимума появилось в конце 19 в. в работах Г. Ф. Вороного [1] и Г. Минковского [2], а многогранники Клейна - в работе $\Phi$. Клейна [3] в связи с обобщением алгоритма непрерывных дробей на многомерный случай. Обе конструкции мотивированы теоремой Лагранжа о наилучших приближениях. Так, например, если $\alpha \in(0,1 / 2)$, решетка $\Gamma_{\alpha}$ порождена векторами $(1, \alpha),(0,1)$, то

$$
\mathfrak{M}\left(\Gamma_{\alpha}\right)=\mathscr{V}\left(\Gamma_{\alpha}\right)=\left\{ \pm\left(Q_{i}, \alpha Q_{i}-P_{i}\right): i=-1,0,1,2, \ldots\right\}
$$

где $Q_{-1}=0, P_{-1}=1$ и $P_{i} / Q_{i}-i$-я подходящая дробь к $\alpha(i \geqslant 0)$.

Отметим, что начиная с размерности $s=3$ множества $\mathfrak{M}(\Gamma)$ и $\mathscr{V}(\Gamma)$ существенно отличаются, хотя известно, что [4]

$$
\mathscr{V}(\Gamma) \subset \mathfrak{M}(\Gamma)
$$

Относительные минимумы и многогранники Клейна возникают в самых различных областях математики (см., например, [5]-[13] и цитированную там литературу). В. И. Арнольдом был сформулирован ряд задач о геометрических и статистических свойствах многомерных непрерывных дробей [5]-[7]. За последние 20 лет появилось большое число работ, посвященных исследованию многогранников Клейна и относительных минимумов. Однако несмотря на значительный интерес, довольно мало известно о статистических свойствах полиэдров Клейна решеток размерности три и выше (в двумерном случае соответствующие результаты вытекают из теории непрерывных дробей). В [8] приведены 
некоторые результаты о существовании статистик многогранников Клейна, в диссертации [9] некоторые из этих статистик приближенно вычислены. В [10] сформулированы гипотезы о вычислении вероятности появления многоугольников заданного типа в качестве грани многогранника Клейна. В [14] получены двусторонние оценки

$$
\frac{1}{\# \mathscr{L}_{s}(\mathbb{Z} ; N)} \sum_{\Gamma \in \mathscr{L}_{s}(\mathbb{Z} ; N)} \# \mathscr{V}(\Gamma) \asymp \ln ^{s-1} N+1
$$

для среднего количества вершин многогранников Клейна целочисленных $s$-мерных решеток с определителем $N$.

В работах [15]-[17] были получены асимптотические формулы для среднего количества относительных минимумов многомерных целочисленных решеток. В настоящей статье с помощью методов, развитых в [15]-[17], выводятся формулы для средних значений количества вершин и граней фиксированного типа полиэдров Клейна трехмерных решеток. Чтобы сформулировать результаты, введем некоторые определения и обозначения.

Пусть $F_{1}$ и $F_{2}$ - многоугольники из $\mathbb{R}^{3}$ с вершинами в $\mathbb{Z}^{3}$. Напомним, что $F_{1}$ и $F_{2}$ принадлежат одному целочисленно-линейному типу, если существует отображение $L \in \mathrm{GL}_{3}(\mathbb{Z})$ такое, что $L F_{1}=F_{2}$.

Возьмем любую компактную грань $F$ многогранника Клейна решетки $\Gamma \in \mathscr{L}_{3}(\mathbb{R})$. Здесь и далее под гранями понимаем двумерные грани (т.е. гиперграни). Пусть $L: \mathbb{R}^{3} \rightarrow \mathbb{R}^{3}$ такое линейное преобразование, что

$$
L \Gamma=\mathbb{Z}^{3} .
$$

Нетрудно заметить, что целочисленно-линейный тип многоугольника $L F$ не зависит от выбора $L$. Это позволяет следующим образом классифицировать грани полиэдров Клейна.

Пусть $\mathscr{T}$ - целочисленно-линейный тип многоугольников из $\mathbb{R}^{3}$.

ОПРЕДЕЛЕНИЕ 0.1. Будем говорить, что грань $F$ принадлежит muny $\mathscr{T}$, если $L F$ принадлежит целочисленно-линейному типу $\mathscr{T}$ для любого линейного преобразования $L: \mathbb{R}^{3} \rightarrow \mathbb{R}^{3}$, удовлетворяющего $(0.2)$.

ЗАмЕчание 0.1 . Любую трехмерную решетку $Г$ можно записать в виде $\Gamma=$ $M \cdot \mathbb{Z}^{3}$, где $M$ - базисная матрица $Г$. Поэтому линейное преобразование $L$ удовлетворяет (0.2) тогда и только тогда, когда $L x=M^{-1} \cdot x$, где $M$ - некоторая базисная матрица решетки Г.

Пусть $\mathscr{F}(\Gamma ; \mathscr{T})$ - множество граней типа $\mathscr{T}$ многогранников Клейна решетки Г. Определим $T F_{3}-$ множество типов компактных граней, которые реализуются на решетках из $\mathscr{L}_{3}(\mathbb{R})$, т.е.

$$
\forall \mathscr{T} \in T F_{3} \quad \exists \Gamma \in \mathscr{L}_{3}(\mathbb{R}): \quad \mathscr{F}(\Gamma, \mathscr{T}) \neq \varnothing .
$$

Пусть

$$
E_{\mathscr{T}}(N)=\frac{1}{\# \mathscr{L}_{3}(\mathbb{Z} ; N)} \sum_{\Gamma \in \mathscr{L}_{3}(\mathbb{Z} ; N)} \# \mathscr{F}(\Gamma, \mathscr{T})
$$


- среднее количество граней типа $\mathscr{T}$ и

$$
E_{\mathscr{V}}(N)=\frac{1}{\# \mathscr{L}_{3}(\mathbb{Z} ; N)} \sum_{\Gamma \in \mathscr{L}_{3}(\mathbb{Z} ; N)} \# \mathscr{V}(\Gamma)
$$

- среднее количество вершин многогранников Клейна трехмерных целочисленных решеток с определителем $N$.

Основным результатом настоящей работы являются следующие асимптотические формулы:

$$
\begin{gathered}
E_{\mathscr{T}}(N)=\mathscr{C}_{\mathscr{T}} \cdot \ln ^{2} N+O_{\mathscr{T}}(\ln N \cdot \ln \ln N), \\
E_{\mathscr{V}}(N)=\mathscr{C}_{\mathscr{V}} \cdot \ln ^{2} N+O(\ln N \cdot \ln \ln N),
\end{gathered}
$$

которые справедливы для всех натуральных $N>2$ и $\mathscr{T} \in T F_{3}$. Здесь $\mathscr{C}_{\mathscr{V}}-$ абсолютная положительная постоянная, константа $\mathscr{C}_{\mathscr{T}}>0$ зависит только от типа $\mathscr{T}$. Аналитические выражения для них довольно громоздкие и приводятся ниже.

Доказательство основано на параметризации соответствующих множеств семействами целочисленных матриц с определителем $N$ и применении результата из [17] (см. также [16]) о количестве целочисленных матриц с фиксированным определителем, лежащих в заданном множестве. Для подсчета вершин также используются вложение (0.1), результаты О.Н. Германа [18] о характеризации относительных минимумов, не являющихся вершинами многогранников Клейна, и О.Н. Карпенкова [11] о классификации граней.

Результаты работы были частично анонсированы в [19].

Автор благодарен В. А. Быковскому за внимание к работе и полезные советы, а также рецензенту за указанные ошибки в первоначальной версии статьи.

\section{§ 1. О количестве целочисленных матриц в заданной области}

Конусом в $\mathbb{R}^{s}$ будем называть множество $V \subset \mathbb{R}^{s}$, удовлетворяющее условию $\lambda x \in V$ при $\lambda \in \mathbb{R}_{+}=(0,+\infty), x \in V$.

Гиперповерхность $S \subset \mathbb{R}^{s}$ называем кусочно дифферениируемой, если она состоит из фиксированного числа гиперповерхностей класса $C^{1}$.

Элемент матрицы $X$, лежащий в $i$-й строке и $j$-м столбце, обозначаем через $x_{i j}$.

Пусть множество $\Omega \subset \mathrm{GL}_{s}(\mathbb{R})$ удовлетворяет следующим условиям:

(A) $\Omega=\left\{X:\left(x_{i 1}, \ldots, x_{i s}\right) \in V_{i}, i=\overline{1, s}\right\}$, где $V_{1}, \ldots, V_{s}$ - конусы из $\mathbb{R}^{s}$ с кусочно дифференцируемыми границами;

(Б) существует такая постоянная $C>0$, что для любой $X \in \Omega$

$$
\prod_{i=1}^{s} \max _{1 \leqslant j \leqslant s}\left|x_{i j}\right| \leqslant C \cdot \operatorname{det} X .
$$

Из условия (А), в частности, следует, что множество $\Omega$ инвариантно относительно левого действия группы $\mathscr{D}_{s}\left(\mathbb{R}_{+}\right)$, состоящей из диагональных матриц $X \in \mathrm{GL}_{s}(\mathbb{R})$ с положительными элементами на главной диагонали. 
Рассмотрим $\left(s^{2}-s\right)$-мерное многообразие $\mathrm{PGL}_{s}(\mathbb{R})=\mathscr{D}_{s}\left(\mathbb{R}_{+}\right) \backslash \mathrm{GL}_{s}(\mathbb{R})$ проективизация группы $\mathrm{GL}_{s}(\mathbb{R})$ относительно левого действия группы $\mathscr{D}_{s}\left(\mathbb{R}_{+}\right)$.

Пусть $\mathscr{P}(\Omega)$ - образ множества $\Omega \subset \mathrm{GL}_{s}(\mathbb{R})$ при проективизации $\mathrm{GL}_{s}(\mathbb{R}) \rightarrow$ $\mathrm{PGL}_{s}(\mathbb{R})$. Для любой перестановки $k=\left(k_{1}, \ldots, k_{s}\right)$ из $\{1, \ldots, s\}$ и набора $\theta=$ $\left(\theta_{1}, \ldots, \theta_{s}\right), \theta_{i}= \pm 1$, определим множества

$$
\begin{gathered}
\mathrm{GL}_{s}(\mathbb{R}, k, \theta)=\left\{X \in \mathrm{GL}_{s}(\mathbb{R}): x_{i k_{i}}=\theta_{i}, i=\overline{1, s}\right\} \\
\mathrm{PGL}_{s}(\mathbb{R}, k, \theta)=\mathscr{P}\left(\mathrm{GL}_{s}(\mathbb{R}, k, \theta)\right) .
\end{gathered}
$$

Тогда $\mathrm{PGL}_{s}(\mathbb{R})$ содержится в объединении всех $\mathrm{PGL}_{s}(\mathbb{R}, k, \theta)$, причем каждый элемент из $\mathrm{PGL}_{s}(\mathbb{R}, k, \theta)$ имеет единственный прообраз при проектировании

$$
\mathrm{GL}_{s}(\mathbb{R}, k, \theta) \rightarrow \mathrm{PGL}_{s}(\mathbb{R}, k, \theta)
$$

Значит, множество всех $\mathrm{PGL}_{s}(\mathbb{R}, k, \theta)$ образует атлас многообразия $\mathrm{PGL}_{s}(\mathbb{R})$, а матрицы из $\mathrm{GL}_{s}(\mathbb{R}, k, \theta)$ являются координатами соответствующих элементов $\mathrm{PGL}_{s}(\mathbb{R}, k, \theta)$.

Определим меру $\widetilde{\mu}=\widetilde{\mu}_{k, \theta}$ на $\mathrm{GL}_{s}(\mathbb{R}, k, \theta)$ следующим образом:

$$
\widetilde{\mu}(W)=\int_{W} \frac{d X}{|\operatorname{det} X|^{s}} \quad \text { при } W \subset \mathrm{GL}_{s}(\mathbb{R}, k, \theta),
$$

где $d X$ - дифференциал $\left(s^{2}-s\right)$-мерной меры Лебега поверхности $\mathrm{GL}_{s}(\mathbb{R}, k, \theta)$ в точке $X$. Она естественным образом порождает меру $\mu_{P}$ на карте $\mathrm{PGL}_{s}(\mathbb{R}, k, \theta)$ :

$$
\mu_{P}(w)=\widetilde{\mu}(W) \quad \text { при } w \subset \mathrm{PGL}_{s}(\mathbb{R}, k, \theta),
$$

где $W$ - прообраз $w$ при проектировании (1.1).

Нетрудно проверить, что мера $\mu_{P}$ не зависит от выбора карты. Поэтому $\mu_{P}$ корректно определена на всем многообразии $\mathrm{PGL}_{s}(\mathbb{R})$.

Если $\Omega$ измеримо по Лебегу и удовлетворяет условию (Б), то множество $\mathscr{P}(\Omega)$ является $\mu_{P}$-измеримым.

Определим также

$$
\mu(\Omega)=\mu_{P}(\mathscr{P}(\Omega))
$$

при $\Omega \subset \mathrm{GL}_{s}(\mathbb{R})$ (если $\Omega \subset \mathrm{GL}_{s}(\mathbb{R}, k, \theta)$, то $\mu(\Omega)=\widetilde{\mu}_{k, \theta}(\Omega)$ ).

Отображение $\mu$ инвариантно относительно правого действия группы $\mathrm{GL}_{s}(\mathbb{R})$, т.е.

$$
\mu(\Omega \cdot Q)=\mu(\Omega) \quad \text { при } Q \in \mathrm{GL}_{s}(\mathbb{R}), \quad \Omega \subset \mathrm{GL}_{s}(\mathbb{R}) .
$$

Эта формула легко проверяется, если матрица $Q$ удовлетворяет одному из следующих условий:

1) $Q$ - диагональная матрица;

2) для любого $X \in \mathrm{GL}_{s}(\mathbb{R})$ матрица $X \cdot Q$ получается из матрицы $X$ изменением нумерации столбцов;

3) для любого $X \in \mathrm{GL}_{s}(\mathbb{R})$ матрица $X \cdot Q$ получается из матрицы $X$ заменой одного из столбцов матрицы $X$ на его сумму с некоторым другим столбцом. 
В общем случае достаточно заметить, что любую невырожденную матрицу можно представить в виде произведения матриц вида 1), 2), 3).

Для любого натурального $N$ определим

$$
\chi(N)=1+\sum_{p \mid N} \frac{\ln p}{p}, \quad \text { где суммирование ведется по простым делителям } N .
$$

Стандартным образом доказывается, что для любого $N \geqslant 3$

$$
\chi(N) \ll 1+\ln \omega(N) \ll 1+\ln \ln N,
$$

где $\omega(N)$ - количество простых делителей $N$.

Теорема 1.1. Пусть множество $\Omega \subset \mathrm{GL}_{s}(\mathbb{R})$ удовлетворяет условиям (А), (Б). Тогда для любого и,лого $N>1$ количество иелочисленных матрии, $M \in \Omega c \operatorname{det} M=N$ равно

$$
\frac{\# \mathscr{L}_{s}(\mathbb{Z} ; N)}{(s-1) !}\left(\frac{\mu(\Omega)}{\zeta(2) \cdots \zeta(s)} \cdot \ln ^{s-1} N+O_{\Omega}\left(\chi(N) \cdot \ln ^{s-2} N\right)\right) .
$$

Доказательство теоремы приведено в [17].

ЗАмЕчАНИЕ 1.1. Пусть множество $\Omega \subset \mathrm{GL}_{s}(\mathbb{R})$ удовлетворяет $(\mathrm{A})$ и следующему условию:

$$
\exists C>0: \quad \forall X \in \Omega \quad \prod_{i=1}^{s} \max _{1 \leqslant j \leqslant s}\left|x_{i j}\right| \leqslant C \cdot|\operatorname{det} X| .
$$

(В отличие от (Б) в правой части неравенства стоит $|\operatorname{det} X|$ вместо $\operatorname{det} X)$. Тогда количество целочисленных матриц $M \in \Omega$ с $|\operatorname{det} M|=N$ также равно (1.2). Для доказательства достаточно заметить, что множества $\Omega^{+}=\{X \in \Omega: \operatorname{det} X \geqslant 0\}$ и $\Omega^{-}=\{X \in \Omega: \operatorname{det} X \leqslant 0\}$ не пересекаются и применить теорему 1.1 .

СлеДСТвИЕ 1.1. Пусть $s=3$ и множество $\Omega \subset \mathrm{GL}_{3}(\mathbb{R})$ удовлетворяет условиям $(\mathrm{A}),\left(\mathrm{Б}^{\prime}\right)$. Пусть $d \in \mathbb{N}, d_{2}, d_{3} \in \mathbb{Z}$. Тогда для любого иелого $N>1$ количество иелочисленных матрии, $M=M(a, b, c) \in \Omega c|\operatorname{det} M|=d N$, удовлетворяющих условиям

$$
\left(a+d_{2} b+d_{3} c\right) \in d \cdot \mathbb{Z}^{3},
$$

равно

$$
\frac{\# \mathscr{L}_{3}(\mathbb{Z} ; N)}{2}\left(\frac{\mu(\Omega)}{\zeta(2) \zeta(3)} \cdot \ln ^{2} N+O_{\Omega, d_{2}, d_{3}, d}(\chi(N) \cdot \ln N)\right) .
$$

ДокАЗАТЕЛЬСтво. Определим

$$
G=\left(\begin{array}{ccc}
d^{-1} & 0 & 0 \\
d_{2} d^{-1} & 1 & 0 \\
d_{3} d^{-1} & 0 & 1
\end{array}\right)
$$

Тогда количество искомых матриц равно количеству целочисленных матриц с определителем $N$, лежащих в $\Omega^{\prime}=\Omega \cdot G$. Нетрудно увидеть, что множество $\Omega^{\prime}$ удовлетворяет условиям $(\mathrm{A}),\left(\mathrm{Б}^{\prime}\right)$. Осталось применить теорему 1.1 , замечание 1.1 и учесть, что $\mu\left(\Omega^{\prime}\right)=\mu(\Omega \cdot G)=\mu(\Omega)$. 


\section{§ 2. Количество граней фиксированного типа}

Целью параграфа является доказательство асимптотической формулы (0.3).

ОПРЕДЕлЕниЕ 2.1. Многоугольник (многогранник) с вершинами в узлах решетки Г будем называть пустым относителъно Г, если он не содержит точек Г, кроме своих вершин.

ОПРЕДЕЛЕНиЕ 2.2. Пирамиду (с отмеченной вершиной) с вершинами в узлах решетки Г будем называть почти пустой относительно $Г$, если она не содержит точек Г, отличных от вершины и точек основания.

Если $\Gamma=\mathbb{Z}^{s}$, то фразу “относительно $\Gamma$ ” в определениях 2.1, 2.2 опускаем.

Введем еще одно важное обозначение. Пусть $\Gamma \in \mathscr{L}_{3}(\mathbb{R})$, плоскость $\pi$ содержит хотя бы три линейно независимых узла решетки Г. Возьмем любые три линейно независимых узла $a, b, c \in \Gamma \cap \pi$ такие, что треугольник с вершинами в точках $a, b, c$ является пустым относительно Г. Обозначим через

$$
d(\Gamma ; \pi)=\frac{|\operatorname{det} M(a, b, c)|}{\operatorname{det} \Gamma}
$$

индекс подрешетки, порожденной $a, b, c$. Если $F$ - грань многогранника Клейна решетки $\Gamma$, содержащаяся в плоскости $\pi_{F}$, то полагаем

$$
d(\Gamma ; F)=d\left(\Gamma ; \pi_{F}\right) .
$$

ЗАмечАниЕ 2.1. Если $M$ - базисная матрица $\Gamma$, то величина $d\left(\Gamma ; \pi_{F}\right)$ совпадает с целочисленным расстоянием от начала координат до плоскости $M^{-1} \cdot \pi_{F}$. Значит, $d(\Gamma ; F)$ однозначно определяется типом грани.

Минимальная окрестность грани. Пусть $F$ - многоугольник из $[0,+\infty)^{3}$ с вершинами в узлах решетки $\Gamma \in \mathscr{L}_{3}(\mathbb{R})$. Через $\pi_{F}$ обозначим плоскость, проходящую через $F$. Она выделяет в $\mathbb{R}^{3}$ два открытых полупространства, которые мы обозначим $S_{F}^{+}$и $S_{F}^{-}$так, что $S_{F}^{-}$содержит начало координат. Из определения вытекает, что $F$ является гранью многогранника Клейна решетки $\Gamma$, если и только если любой ненулевой узел $Г$ из $S_{F}^{-} \cup \pi_{F} \backslash F$ имеет хотя бы одну отрицательную координату. Докажем, что достаточно рассматривать некоторое конечное множество узлов.

Определим множество $X=X(\Gamma ; F)$ следующим образом. Пусть $d=d\left(\Gamma ; \pi_{F}\right)$. Если $d=1$, то полагаем $X=\pi_{F} \cap \Gamma$. Пусть $d>1$. Все точки решетки $\Gamma$ лежат на плоскостях, параллельных $\pi_{F}$, которые расположены на одинаковом расстоянии друг от друга. Занумеруем такие плоскости, лежащие в $S_{F}^{-}$, через $\pi_{d-1}, \pi_{d-2}, \ldots$ по возрастанию расстояния до $\pi_{d}=\pi_{F}$. Тогда $\pi_{0}$ проходит через начало координат. Положим

$$
X=\bigcup_{r=1}^{d} \frac{d}{r} \cdot\left(\Gamma \cap \pi_{r}\right),
$$

т.е. $X$ состоит из узлов $\gamma \in \Gamma \cap \pi_{F}$ и точек пересечения $\pi_{F}$ с прямыми, проходящими через начало координат и узлы решетки $\Gamma$, лежащие между плоскостями $\pi_{0}$ и $\pi_{F}=\pi_{d}$. 
ОПРЕДЕЛЕниЕ 2.3. Если $X$ - дискретное, а $F$ - выпуклое замкнутое множество из $\mathbb{R}^{s}$, то множество

$$
P(X, F)=\{u \in X \backslash F:(\operatorname{Conv}(F \cup\{u\}) \backslash F) \cap X=\{u\}\},
$$

будем называть минимальной $X$-окрестностью $F$.

Лемма 2.1. Пусть $F$ - многоугольник из $[0,+\infty)^{3}$ с вериинами в узлах решетки $\Gamma \in \mathscr{L}_{3}(\mathbb{R}), X=X(\Gamma ; F)$ и $P=P(X ; F)$ - минимальная $X$-окрестность $F$.

Тогда F является гранъю многогранника Клейна решетки Г, если и только если выполняются следующие условия:

$$
P \cap[0,+\infty)^{3}=\varnothing,
$$

пирамида $\operatorname{Conv}(F \cup\{0\})$ с основанием $F$ почти пустая относительно $Г$.

ДокАЗАтЕльство. Пусть $F$ является гранью многогранника Клейна. Тогда любой ненулевой узел $\Gamma$ из $\left(S_{F}^{-} \cup \pi_{F}\right) \backslash F$ имеет хотя бы одну отрицательную координату и, значит, выполняются $(2.1),(2.2)$.

Пусть выполняются условия $(2.1),(2.2), d=d(\Gamma ; F), \pi_{F}=\pi_{d}$ - плоскость, проходящая через $F$. Докажем, что $F$ является гранью полиэдра Клейна решетки $Г$. Возьмем любой ненулевой узел $u \in \Gamma \cap\left(S_{F}^{-} \cup \pi_{F}\right) \backslash F$. Нужно показать, что $u \notin[0,+\infty)^{3}$. Имеем $u \in \pi_{r}$ c $r \leqslant d$. Отдельно рассмотрим два случая.

1. Пусть $1 \leqslant r \leqslant d$. Тогда $\widetilde{u}=(d / r) \cdot u \in X$. Согласно $(2.2) \widetilde{u} \notin F$. Значит, существует точка $v \in P$, принадлежащая множеству $\operatorname{Conv}(F \cup\{\widetilde{u}\})$. Если $u \in[0,+\infty)^{3}$, то $\widetilde{u} \in[0,+\infty)^{3}$ и $v \in[0,+\infty)^{3}$. Получили противоречие с $(2.1)$.

2. Пусть $r \leqslant 0$. Из вышедоказанного вытекает, что

$$
\Gamma \cap \pi_{F} \cap[0,+\infty)^{3}=\Gamma \cap F .
$$

Это возможно только в случае, когда плоскость $\pi_{F}$ пересекает координатные оси в точках вида $\left(\theta_{1}, 0,0\right),\left(0, \theta_{2}, 0\right),\left(0,0, \theta_{1}\right)$, где $\theta_{i}>0$. Тогда любая из плоскостей $\pi_{r}$ c $r \leqslant 0$ не содержит точек из $[0,+\infty)^{3} \backslash\{0\}$. Значит, $u \notin[0,+\infty)^{3}$.

\section{Конечность минимальной окрестности.}

Лемма 2.2. Пусть $t \in(1,+\infty), \Gamma^{\prime} \in \mathscr{L}_{2}(\mathbb{R})$. Возъмем любой треугольник $\Delta \subset \mathbb{R}^{2}$ такой, что две вершины $\Delta$ принадлежат $\Gamma^{\prime}$, а оставшаяся содержится в $t \Gamma^{\prime}$ и $\Delta$ не содержит других точек множества $\Gamma^{\prime} \cup\left(t \Gamma^{\prime}\right)$. Тогда

$$
\operatorname{mes} \Delta \leqslant \frac{[t]+1}{2} \cdot \operatorname{det} \Gamma^{\prime}
$$

где $[t]-$ челая часть $t$.

ДокАзАтЕЛьство. Обозначим вершины $\Delta$ через $a, b, c$ так, что

$$
a, b \in \Gamma^{\prime}, \quad c \in t \Gamma^{\prime} .
$$

Пусть $W$ - полоса, ограниченная прямыми $l_{1}, l_{2}$, где $l_{1}\left(l_{2}\right)$ проходит через точку $a$ (точку $b)$ параллельно отрезку $[c, b]$. При этом $l_{1} \cap W=\varnothing, l_{2} \subset W$. 
Пусть $W_{+}-$"половина" полосы $W$, состоящая из точек $x \in W$ таких, что $x$ и $b$ лежат по разные стороны от прямой, проходящей через $a$ и $c$.

Так как $a, b \in \Gamma^{\prime}$, то $W_{+}$содержит бесконечно много точек решетки $\Gamma^{\prime}$. Возьмем любой узел $d \in \Gamma^{\prime} \cap W_{+}$такой, что треугольник $\Delta_{a b d}$ (с вершинами в точках $a, b, d)$ пустой относительно $\Gamma^{\prime}$. Рассмотрим точки $d_{k}=a+k(d-a), k \in \mathbb{N}$. Они лежат на прямой, проходящей через $d, a$. Пусть $n$ - наименьшее натуральное число такое, что отрезок $\left[a, d_{n}\right]$ пересекается с прямой, проходящей через точку $c$ параллельно $[a, b]$. Рассмотрим треугольник $\Delta_{n}$ с вершинами в $a, b, d_{n}$. Имеем

$$
\operatorname{mes} \Delta \leqslant \operatorname{mes} \Delta_{n}=n \cdot \operatorname{mes} \Delta_{a b d}=n \cdot \frac{\operatorname{det} \Gamma^{\prime}}{2} \text {. }
$$

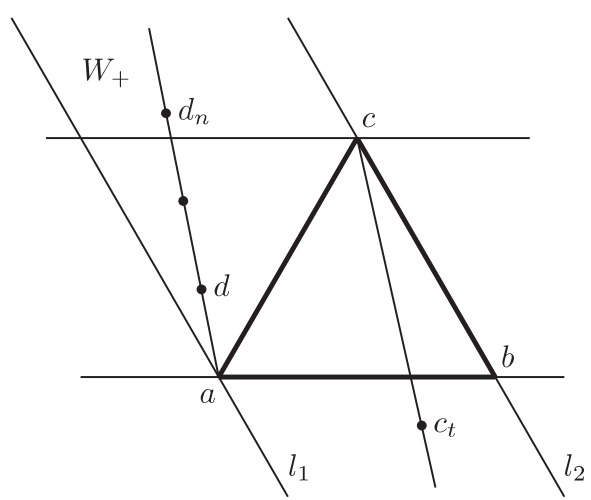

Рис. 1

Осталось оценить $n$. Рассмотрим точку

$$
c_{t}=c-t(d-a) \in t \Gamma^{\prime}
$$

Если $t \leqslant n-1$, то $c_{t}$ принадлежит треугольнику $\Delta$ и не совпадает с его вершинами. Это противоречит условиям леммы. Значит, $n<t+1$, и поэтому

$$
\operatorname{mes} \Delta \leqslant n \cdot \frac{\operatorname{det} \Gamma^{\prime}}{2} \leqslant([t]+1) \cdot \frac{\operatorname{det} \Gamma^{\prime}}{2} .
$$

СледСтвие 2.1. Пусть $t_{1}=1 u t_{i} \in(1,+\infty), i=\overline{2, d}$. Определим

$$
X=\bigcup_{i=1}^{d}\left(t_{i} \mathbb{Z}^{2}\right) .
$$

Пусть $F$ - выпуклый многоугольник с вершинами из $\mathbb{Z}^{2}$, причем $F \cap X=F \cap \mathbb{Z}^{2}$. Тогда минимальная $X$-окрестность $F$ является конечным множеством.

ДоказАтельство. Пусть $P=P(X ; F)$ - минимальная $X$-окрестность $F$. Достаточно доказать, что множество $P$ ограниченное. 
Пусть $B$ - произвольный круг, содержащийся в $F$ с центром в точке $x_{0}$ радиуса $R>0$. Возьмем любую точку $x \in P$ и положим

$$
U_{F}=\operatorname{Conv}(F \cup\{x\}), \quad U_{B}=\operatorname{Conv}(B \cup\{u\}) .
$$

Тогда $U_{B} \subset U_{F}$. Кроме того, $x \in t_{j} \mathbb{Z}^{2}$ с некоторым $j \in\{1, \ldots, d\}$. Пусть $r=\#\left(\mathbb{Z}^{2} \cap \partial F\right)$. Рассмотрим многоугольник $U_{F} \backslash F$. Он состоит из не более чем $r$ треугольников $\Delta$, каждый из которых удовлетворяет следующим условиям: 1 ) $x$ - вершина $\Delta ; 2)$ две остальные вершины $\Delta$ являются точками $F \cap X$ и поэтому принадлежат $\left.\mathbb{Z}^{2} ; 3\right)$ треугольник $\Delta$ не содержит других точек множества $\mathbb{Z}^{2} \cup$ $\left(t_{j} \mathbb{Z}^{2}\right)$. Согласно лемме 2.2 площадь каждого такого треугольника не больше чем $\left[t_{j}+1\right] / 2$. Значит, $\operatorname{mes}\left(U_{F} \backslash F\right) \leqslant r\left[t_{j}+1\right] / 2$, поэтому

$$
R\left|x-x_{0}\right|+\frac{\pi R^{2}}{2}=\operatorname{mes} U_{B} \leqslant \operatorname{mes} U_{F} \leqslant \operatorname{mes} F+\frac{r\left[t_{j}+1\right]}{2} .
$$

Множество $P$ ограниченное. Лемма доказана.

Параметризация граней. Возьмем любой тип $\mathscr{T} \in T F_{3}$. Зафиксируем любой многоугольник $T \in \mathscr{T}$, который параллелен плоскости $x_{3}=0$, а также упорядоченную тройку целочисленных точек $(\alpha, \beta, \gamma)$ таких, что треугольник с вершинами в точках $\alpha, \beta+\alpha, \gamma+\alpha$ содержится в $T$ и является пустым, т.е.

$$
\mathbb{Z}^{3} \cap \operatorname{Conv}(\alpha, \alpha+\beta, \alpha+\gamma)=\{\alpha, \alpha+\beta, \alpha+\gamma\} \subset T .
$$

Не ограничивая общности, считаем, что $\operatorname{det} M(\alpha, \beta, \gamma)>0$. Пусть $d_{1}, d_{2}, d_{3}-$ алгебраические дополнения к элементам последней строки матрицы $M(\alpha, \beta, \gamma)$,

$$
\widetilde{\alpha}=\frac{\alpha+d_{2} \beta+d_{3} \gamma}{d}
$$

Нетрудно заметить, что

$$
M(\alpha, \beta, \gamma)=\left(\begin{array}{ccc}
\alpha_{1} & \beta_{1} & \gamma_{1} \\
\alpha_{2} & \beta_{2} & \gamma_{2} \\
d & 0 & 0
\end{array}\right), \quad d_{1}=1,
$$

где $d=d(\mathscr{T})$ - целочисленное расстояние от плоскости, содержащей многоугольник типа $\mathscr{T}$, до начала координат. Поэтому $\widetilde{\alpha}=(0,0,1)$ и тройка $\{\widetilde{\alpha}, \beta, \gamma\}$ образует базис решетки $\mathbb{Z}^{3}$.

Так как $T, \alpha, \beta, \gamma$ фиксированы, то далее пишем

$$
T=T(\mathscr{T}), \quad(\alpha, \beta, \gamma)=(\alpha, \beta, \gamma)(\mathscr{T}), \quad d_{2}=d_{2}(\mathscr{T}), \quad d_{3}=d_{3}(\mathscr{T}),
$$

имея в виду, что эти объекты однозначно определяются по типу $\mathscr{T}$.

Определим множество

$$
X(\mathscr{T})=\bigcup_{r=1}^{d} \frac{d}{r} \cdot \mathbb{Z}_{r}^{3}
$$

где $\mathbb{Z}_{r}^{3}$ - множество точек из $\mathbb{Z}^{3}$, у которых третья координата равна $r$. 
Так как $\widetilde{\alpha}, \beta, \gamma$ образуют базис $\mathbb{Z}^{3}$, причем $\widetilde{\alpha}_{3}=1, \beta_{3}=\gamma_{3}=0$, то $X(\mathscr{T})$ состоит из точек $x(r, n, k)$ следующего вида:

$$
x(r, n, k)=d \cdot\left(\widetilde{\alpha}+\frac{n}{r} \cdot \beta+\frac{k}{r} \cdot \gamma\right), \quad r \in \mathbb{Z} \cap[1, d], \quad n, k \in \mathbb{Z} .
$$

Пусть $P(\mathscr{T})$ - минимальная $X(\mathscr{T})$-окрестность множества $T$. Так как $\mathscr{T} \in T F_{3}$, то пирамида с основанием $T$ и вершиной в начале координат является почти пустой. Поэтому $X(\mathscr{T}) \cap T=\mathbb{Z}^{3} \cap T$ и, используя лемму 2.2 , получаем

$$
\# P(\mathscr{T})=O_{\mathscr{T}}(1) .
$$

Пусть множества $J(\mathscr{T}) \subset \mathbb{Z}^{2}, J_{P}(\mathscr{T}) \subset \mathbb{Z}^{3}$ такие, что

$$
\begin{aligned}
\mathbb{Z}^{3} \cap T & =\{\alpha+n \beta+k \gamma:(n, k) \in J(\mathscr{T})\}, \\
P(\mathscr{T}) & =\left\{x(r, n, k):(r, n, k) \in J_{P}(\mathscr{T})\right\} .
\end{aligned}
$$

Возьмем любую решетку $\Gamma \in \mathscr{L}_{3}(\mathbb{R})$ и грань $F \in \mathscr{F}(\Gamma ; \mathscr{T})$, лежащую в угле $[0,+\infty)^{3}$. Согласно определению типа грани существует базисная матрица $M$ решетки $\Gamma$ такая, что $M^{-1} \cdot F=T$. Обозначим через $\Lambda(\Gamma ; F)$ множество упорядоченных троек $(a, b, c) \in \Gamma^{3}$, для каждой из которых существует базисная матрица $M$ решетки $\Gamma$, удовлетворяющая условиям

$$
M^{-1} \cdot F=T, \quad M^{-1} \cdot a=\alpha, \quad M^{-1} \cdot b=\beta, \quad M^{-1} \cdot c=\gamma .
$$

Нетрудно заметить, что величина $\# \Lambda(\Gamma ; F)$ зависит только от $\mathscr{T}$, а именно

$$
\# \Lambda(\Gamma ; F)=\nu(\mathscr{T})
$$

где $\nu(\mathscr{T})$ - количество матриц $S \in \mathrm{GL}_{3}(\mathbb{Z})$ таких, что

$$
S \cdot T=T, \quad S \cdot\{\alpha, \beta, \gamma\}=\{\alpha, \beta, \gamma\} .
$$

Если базисная матрица $M$ решетки Г удовлетворяет (2.5), то

$$
\begin{gathered}
X(\Gamma ; F)=M \cdot X(\mathscr{T}), \\
P(X ; F)=M \cdot P(\mathscr{T})=\left\{d\left(\widetilde{a}+\frac{n}{r} \cdot b+\frac{k}{r} \cdot c\right):(r, n, k) \in J_{P}(\mathscr{T})\right\},
\end{gathered}
$$

где $P(X ; F)$ - минимальная $X(\Gamma ; F)$-окрестность грани $F$.

ЛЕмма 2.3. Следующие утверждения эквивалентны.

(a) $\Gamma \in \mathscr{L}_{3}(\mathbb{R}), F \in \mathscr{F}(\Gamma ; \mathscr{T}), F \subset[0,+\infty)^{3},(a, b, c) \in \Lambda(\Gamma ; F)$.

(б) Вектори $a, b, c \in \mathbb{R}^{3}$ удовлетворяют условиям

$$
\begin{gathered}
F=\operatorname{Conv}\{a+n b+k c:(n, k) \in J(\mathscr{T})\} \subset[0,+\infty)^{3}, \\
\left(\widetilde{a}+\frac{n}{r} \cdot b+\frac{k}{r} \cdot c\right) \notin[0,+\infty)^{3} \quad n p u \quad(r, n, k) \in J_{P}(\mathscr{T}),
\end{gathered}
$$

$\Gamma$ - решетка с базисом $\{\widetilde{a}, b, c\}$, a $\widetilde{a}$ определяется формулой

$$
\widetilde{a}=\frac{a+d_{2} b+d_{3} c}{d} .
$$


ДокАЗАтЕльство. Пусть выполняется (а). Из (2.5), (2.3) вытекает, что $\{\widetilde{a}, b, c\}-$ базис $\Gamma$. Соотношение $(2.8)$ следует из определений множеств $J(\mathscr{T})$, $\Lambda(\Gamma ; F)$ и условия $F \subset[0,+\infty)^{3}$, а $(2.9)$ - из $(2.7)$ и леммы 2.1.

Пусть выполняется (б). Нетрудно заметить, что тогда справедливы соотношения (2.7). Докажем, что $F$ - грань многогранника Клейна решетки Г. Для этого достаточно проверить выполнение условий (2.1), (2.2). Соотношение (2.1) вытекает из (2.7) и (2.9). Докажем (2.2). Пусть $M$ - базисная матрица $\Gamma$. Из определений $F$ и $J(\mathscr{T})$ вытекает, что $M^{-1} \cdot F$ принадлежит целочисленно-линейному типу $\mathscr{T}$. Так как $\mathscr{T} \in T F_{3}$, то пирамида с основанием $M^{-1} \cdot F$ и вершиной в начале координат почти пустая. Значит, выполняется (2.2). Применяя лемму 2.1 , получаем $F \in \mathscr{F}(\Gamma ; \mathscr{T})$. Условие $(a, b, c) \in \Lambda(\Gamma ; F)$ следует из определений $\Gamma, F$ и $J(\mathscr{T})$. Лемма доказана.

ЗАмЕчАние 2.2. Если выполняется условие (а) леммы 2.3 и $\Gamma \in \mathscr{L}_{3}(\mathbb{Z} ; N)$, TO

$$
a, b, c \in \mathbb{Z}^{3}, \quad|\operatorname{det} M(a, b, c)|=d \cdot N, \quad\left(a+d_{2} b+d_{3} c\right) \in d \mathbb{Z}^{3} .
$$

Если выполняется условие (б) и $(2.11)$, то $\Gamma \in \mathscr{L}_{3}(\mathbb{Z} ; N)$.

Определим множество $\mathscr{U}_{+}(N ; \mathscr{T})$, состоящее из пар $(\Gamma, F)$, где $\Gamma \in \mathscr{L}_{3}(\mathbb{Z} ; N)$, a $F \in \mathscr{F}(\Gamma ; \mathscr{T})$, причем $F \subset[0,+\infty)^{3}$. Тогда

$$
E_{\mathscr{T}}(N)=2^{3} \cdot \frac{\# \mathscr{U}_{+}(N ; \mathscr{T})}{\# \mathscr{L}_{3}(\mathbb{Z} ; N)} .
$$

Будем использовать следующие матричные множества:

$$
\begin{gathered}
\Omega_{\mathscr{T}}=\left\{M(a, b, c) \in \mathscr{M}_{3}(\mathbb{R}): \text { выполняются }(2.8),(2.9)\right\} \\
\omega_{\mathscr{T}}(N)=\left\{M(a, b, c) \in \Omega_{\mathscr{T}}: \text { выполняются }(2.11)\right\} .
\end{gathered}
$$

СледСтвиЕ 2.2. Для любых $N \in \mathbb{N}, \mathscr{T} \in T F_{3}$ справедлива формула

$$
\# \mathscr{U}_{+}(N ; \mathscr{T})=\frac{1}{\nu(\mathscr{T})} \cdot \# \omega_{\mathscr{T}}(N)
$$

ДоказАтельство. Для каждой пары $(\Gamma, F) \in \mathscr{U}_{+}(N ; \mathscr{T})$ определим

$$
\widetilde{\Lambda}(\Gamma ; F)=\{M(a, b, c):(a, b, c) \in \Lambda(\Gamma ; F)\} .
$$

Согласно лемме 2.3 и замечанию 2.2

$$
\bigcup_{(\Gamma, F) \in \mathscr{U}_{+}(N ; \mathscr{T})} \tilde{\Lambda}(\Gamma ; F)=\omega_{\mathscr{T}}(N) .
$$

Если $\left(\Gamma_{1}, F_{1}\right) \neq\left(\Gamma_{2}, F_{2}\right),\left(\Gamma_{i}, F_{i}\right) \in \mathscr{U}_{+}(N ; \mathscr{T})$, то $\Lambda\left(\Gamma_{1} ; F_{1}\right) \cap \Lambda\left(\Gamma_{2} ; F_{2}\right)=\varnothing$. Поэтому, учитывая (2.6), получаем

$$
\# \omega_{\mathscr{T}}(N)=\sum_{(\Gamma ; F) \in \mathscr{U}_{+}(N ; \mathscr{T})} \# \widetilde{\Lambda}(\Gamma ; F)=\nu(\mathscr{T}) \cdot \# \mathscr{U}_{+}(N ; \mathscr{T}) .
$$


Вычисление $\# \omega_{\mathscr{T}}(N)$ и окончательная формула. Покажем, что для вычисления $\# \omega_{\mathscr{T}}(N)$ можно применить следствие 1.1. Очевидно, что множество $\Omega=\Omega_{\mathscr{T}}$ удовлетворяет условию $(\mathrm{A})$. Осталось проверить выполнение $\left(\mathrm{Б}^{\prime}\right)$.

Лемма 2.4. Пусть $\Gamma \in \mathscr{L}_{3}(\mathbb{R} ; N)$, минимумы $a, b, c \in \mathfrak{M}(\Gamma) \cap[0,+\infty)^{3}$ являтотся базисом Г, причем

$$
a_{1} \geqslant \max \left\{b_{1}, c_{1}\right\}, \quad b_{2} \geqslant \max \left\{a_{2}, c_{2}\right\}, \quad c_{3} \geqslant \max \left\{a_{3}, b_{3}\right\} .
$$

Тогда $a_{1} b_{2} c_{3} \ll N$.

ДоказАтельство. Если минимум $v \in \mathfrak{M}(\Gamma)$ и ненулевой узел $u \in \Gamma$ имеют неотрицательные координаты, то существует номер $k$ такой, что $u_{k} \geqslant 2 v_{k}$, так как в противном случае узел $u-v$ нарушает минимальность $v$. Поэтому

$$
(2 a-b),(2 a-c),(2 b-a),(2 b-c),(2 c-a),(2 c-b) \notin \mathbb{R}_{+}^{3} .
$$

Возьмем любое $\varepsilon \in(0,1 / 4)$ (независящее от $N)$. Если $a_{1} b_{2} c_{3} \leqslant \varepsilon^{-3} N$, то утверждение леммы выполнено. Пусть $a_{1} b_{2} c_{3}>\varepsilon^{-3} N$. Тогда по теореме Минковского о линейных формах существует ненулевой узел $u \in \Gamma$ такой, что

$$
\left|u_{1}\right|<\varepsilon \cdot a_{1}, \quad\left|u_{2}\right|<\varepsilon \cdot b_{2}, \quad\left|u_{3}\right|<\varepsilon \cdot c_{3} .
$$

Так как $a-$ минимум, то $a_{2}<\left|u_{2}\right|$ либо $a_{3}<\left|u_{3}\right|$. Поэтому

$$
a_{2}<\varepsilon \cdot b_{2} \text { либо } a_{3}<\varepsilon \cdot c_{3} .
$$

Аналогично используя минимальность $b, c$, получаем

$$
\begin{array}{lll}
b_{1}<\varepsilon \cdot a_{1} & \text { либо } & b_{3}<\varepsilon \cdot c_{3}, \\
c_{1}<\varepsilon \cdot a_{1} & \text { либо } & c_{2}<\varepsilon \cdot b_{2} .
\end{array}
$$

Применяя эти неравенства и учитывая (2.14), нетрудно проверить, что

$N=\left|\operatorname{det}\left(\begin{array}{lll}a_{1} & b_{1} & c_{1} \\ a_{2} & b_{2} & c_{2} \\ a_{3} & b_{3} & c_{3}\end{array}\right)\right| \geqslant a_{1} b_{2} c_{3}-a_{3} b_{2} c_{1}-a_{1} b_{3} c_{2}-a_{2} b_{1} c_{3}>\left(\frac{1}{2}-2 \cdot \varepsilon\right) a_{1} b_{2} c_{3}$.

Так как $(1 / 2-2 \cdot \varepsilon)>0$, то лемма доказана.

СлеДСТвИЕ 2.3. Пусть $F$ - гранъ полиэдра Клейна решетки $\Gamma \in \mathscr{L}_{3}(\mathbb{R})$, причем $d=d(\Gamma ; F), S=\#(F \cap \Gamma)$. Тогда

$$
\prod_{i=1}^{3} \max _{u \in F \cap \Gamma}\left|u_{i}\right| \ll d \cdot S \cdot \operatorname{det} \Gamma \text {. }
$$

ДокАзАТЕЛЬство. Не ограничивая общности, считаем, что $F \subset[0,+\infty)^{3}$. Пусть $v^{(1)}, v^{(2)}, v^{(3)}-$ вершины $F$ такие, что

$$
\left|v_{i}^{(i)}\right|=\max _{u \in F \cap \Gamma}\left|u_{i}\right|, \quad i=1,2,3 .
$$

Пусть $\Gamma^{\prime}$ - решетка, порожденная $v^{(1)}, v^{(2)}, v^{(3)}$. Очевидно, что $\operatorname{det} \Gamma^{\prime}=O(S \cdot d$. $\operatorname{det} \Gamma)$. Кроме того, $v^{(i)} \in \mathfrak{M}(\Gamma)$ (в силу $\left.(0.1)\right)$, и поэтому $v^{(i)} \in \mathfrak{M}\left(\Gamma^{\prime}\right)$. Осталось воспользоваться леммой 2.4 , в которой $Г$ заменяем на $\Gamma^{\prime}$. 
Теорема 2.1. Пусть $N \in \mathbb{N}, N \geqslant 2, \mathscr{T} \in T F_{3}$. Справедлива следующая асимптотическая формула для среднего числа граней типа $\mathscr{T}$ :

$$
E_{\mathscr{T}}(N)=\mathscr{C}_{\mathscr{T}} \cdot \ln ^{2} N+O_{\mathscr{T}}(\chi(N) \cdot \ln N),
$$

әде

$$
\mathscr{C}_{\mathscr{T}}=\frac{4}{\nu(\mathscr{T})} \cdot \frac{\mu\left(\Omega_{\mathscr{T}}\right)}{\zeta(2) \zeta(3)}
$$

ДоКАЗАТЕЛЬство. Согласно следствию 2.3 множество $\Omega_{\mathscr{T}}$ удовлетворяет условию (Б') (если $M(a, b, c) \in \Omega_{\mathscr{T}}$, то существуют решетка $\Gamma$ и грань $F \in$ $\mathscr{F}(\Gamma ; \mathscr{T})$ такие, что $a,(b+a),(c+a) \in \Gamma \cap F)$. Все условия следствия 1.1 выполнены. Применяя его, получаем

$$
\# \omega_{\mathscr{T}}(N)=\frac{\# \mathscr{L}_{3}(\mathbb{Z} ; N)}{2 \zeta(2) \zeta(3)}\left(\mu\left(\Omega_{\mathscr{T}}\right) \ln ^{2} N+O_{\Omega_{\mathscr{T}}, d_{2}, d_{3}, d}(\chi(N) \cdot \ln N)\right) .
$$

Осталось воспользоваться (2.12), (2.13). Теорема доказана.

ЗАмечАниЕ 2.3. Нетрудно проверить, что множество $\Omega_{\mathscr{T}}$ имеет непустую внутренность, и поэтому $\mu\left(\Omega_{\mathscr{T}}\right)>0, \mathscr{C}_{\mathscr{T}}>0$. Действительно, так как $\mathscr{T} \in T F_{3}$, то существует решетка $\Gamma \in \mathscr{L}_{3}(\mathbb{R})$ и грань $F \in \mathscr{F}(\Gamma ; \mathscr{T}), F \subset[0,+\infty)^{3}$. Легко заметить, что тогда найдутся решетка общего положения $\Gamma^{*}$ (т.е. $\gamma_{i} \neq 0$ при $i=1,2,3$ для всех $\left.\gamma \in \Gamma^{*} \backslash\{0\}\right)$ и грань $F^{*} \in \mathscr{F}\left(\Gamma^{*} ; \mathscr{T}\right), F^{*} \subset[0,+\infty)^{3}$. Для матрицы $M=M(a, b, c) \in \Lambda\left(\Gamma^{*} ; F^{*}\right)$ все неравенства, входящие в определение $\Omega_{\mathscr{T}}$ будут строгими, т.е. $M$ принадлежит внутренности $\Omega_{\mathscr{T}}$.

ЗАмЕчАниЕ 2.4. Утверждения леммы 2.4 и следствия 2.3 не обобщаются на случай размерности 4 и выше. Следующий пример построен О. Н. Германом. Рассмотрим решетку Г с базисной матрицей

$$
M\left(a^{(1)}, a^{(2)}, a^{(3)}, a^{(4)}\right)=\left(\begin{array}{cccc}
1 & 0 & 0 & \varepsilon \\
0 & 1 & \varepsilon & 0 \\
0 & 0 & \frac{1}{2} & \frac{1}{2}+\varepsilon \\
0 & 0 & \frac{1}{2} & \frac{1}{2}-\varepsilon
\end{array}\right),
$$

где $0<\varepsilon<1 / 2$. Тогда множество $F=\operatorname{Conv}\left\{a^{(i)}\right\}_{i=1}^{4}$ является пустой гранью многогранника Клейна решетки Г. Поэтому $a^{(i)} \in \mathscr{V}(\Gamma) \subset \mathfrak{M}(\Gamma)$. Однако

$$
\operatorname{det} \Gamma=\varepsilon, \quad \prod_{i=1}^{4} \max _{1 \leqslant j \leqslant 4}\left|a_{i}^{(j)}\right|=\frac{1}{4}+\frac{\varepsilon}{2} .
$$

О множестве $T F_{3}$ реализуемых граней. Содержание этого пункта приводится для полноты изложения и нигде не используется.

О.Н. Карпенковым [11] получено полное описание целочисленно-линейных типов $\mathscr{T} \in T F_{3}$ многоугольников, принадлежащих плоскостям, лежащим на целочисленном расстоянии большем чем 1 от начала координат. Рассмотрим остальные типы.

Пусть $T F_{3}(1)$ состоит из $\mathscr{T} \in T F_{3}$ таких, что многоугольники целочисленнолинейного типа $\mathscr{T}$ принадлежат плоскостям, лежащим на единичном целочисленном расстоянии от начала координат. 
Лемма 2.5. Пусть $\mathscr{T}$ - целочисленно-линейный тип выпуклых многоугольников из $\mathbb{R}^{3}$. Тогда $\mathscr{T} \in T F_{3}(1)$, если и толъко если для любого $T \in \mathscr{T}$

а) целочисленное расстояние от плоскости, содержащей $T$, до начала координат равно 1 ;

б) существует треугольник $\Delta$ такой, что $T \subset \Delta, \Delta \cap \mathbb{Z}^{3}=T \cap \mathbb{Z}^{3}$.

ЗАмечАниЕ 2.5. Если условия а), б) выполняются для некоторого $T \in \mathscr{T}$, то они выполняются для всех $T \in \mathscr{T}$.

ДокАзАтельство. Пусть $\mathscr{T} \in T F_{3}(1)$. Тогда существуют решетка $\Gamma \in$ $\mathscr{L}_{3}(\mathbb{R})$ и грань $F \in \mathscr{F}(\Gamma ; \mathscr{T})$. Причем их всегда можно выбрать так, что $F \subset[0,+\infty)^{3}$. Рассмотрим треугольник $\Delta_{\Gamma}$ равный пересечению плоскости, проходящей через $F$, и угла $[0,+\infty)^{3}$. Тогда $\Delta_{\Gamma} \cap \Gamma=F \cap \Gamma$. Возьмем любую базисную матрицу $M$ решетки $\Gamma$. Тогда $M^{-1} \Gamma=\mathbb{Z}^{3}$ и условие б), в котором $T=M^{-1} \cdot F, \Delta=M^{-1} \cdot \Delta_{\Gamma}$ выполнено. Условие а) выполняется по определению.

Пусть выполнены условия а) и б). Докажем, что $\mathscr{T} \in T F_{3}(1)$. Возьмем любой многоугольник $T \in \mathscr{T}$. Пусть $\Delta$ - треугольник из б). Рассмотрим пирамиду $P$ с основанием $\Delta$ и вершиной в начале координат. Согласно а), б) она является почти пустой. Возьмем любое линейное преобразование $L$, которое плоскости, проходящие через стороны треугольника $\Delta$ и начало координат, переводит в координатные плоскости. Тогда $\Gamma=L \mathbb{Z}^{3} \in \mathscr{L}_{3}(\mathbb{R}), F=L T \in \mathscr{F}(\Gamma ; \mathscr{T})$.

\section{§ 3. Количество вершин}

Формулировка основного результата. Пусть

$$
\begin{gathered}
\Omega_{1}=\left\{\left(\begin{array}{ccc}
a_{1} & -b_{1} & -c_{1} \\
a_{2} & b_{2} & -c_{2} \\
a_{3} & -b_{3} & c_{3}
\end{array}\right): a_{1}<b_{1}+c_{1}\right\}, \\
\Omega_{2}=\left\{\left(\begin{array}{ccc}
a_{1} & -b_{1} & -c_{1} \\
a_{2} & b_{2} & c_{2} \\
a_{3} & -b_{3} & c_{3}>c_{2}
\end{array}\right): \begin{array}{c}
\text { либо } \\
c_{1}>b_{1}
\end{array}\right\}, \quad \Omega_{3}=\left\{\left(\begin{array}{ccc}
a_{1} & -b_{1} & -c_{1} \\
a_{2} & b_{2} & -c_{2} \\
a_{3} & b_{3} & c_{3}
\end{array}\right)\right\}, \\
\Omega_{4}=\left\{\left(\begin{array}{ccc}
a_{1} & -b_{1} & c_{1} \\
a_{2} & b_{2} & -c_{2} \\
a_{3} & b_{3} & c_{3}
\end{array}\right): \begin{array}{c}
b_{2}<a_{2}+c_{2}, \\
b_{1}>c_{1}
\end{array}\right\},
\end{gathered}
$$

где

$$
\begin{gathered}
a_{i}, b_{i}, c_{i} \in(0,+\infty), \quad i=1,2,3 ; \\
a_{1} \geqslant \max \left\{b_{1}, c_{1}\right\}, \quad b_{2}>\max \left\{a_{2}, c_{2}\right\}, \quad c_{3}>a_{3} \geqslant b_{3} .
\end{gathered}
$$

Кроме того, определим:

$\Omega_{V}$ - множество матриц из $\Omega_{1}$, у которых

$$
b_{2} \leqslant a_{2}+c_{2}, \quad c_{3} \leqslant a_{3}+b_{3}, \quad a_{2} \geqslant c_{2} ;
$$


$\Omega_{V}^{\prime}-$ множество матриц из $\Omega_{V}$, у которых

$$
\begin{array}{rll}
b_{2}>\frac{a_{2}}{2}+c_{2} & \text { либо } & c_{3}>\frac{a_{3}}{2}+b_{3}, \\
a_{1}<2 c_{1} & \text { либо } & a_{2}<2 c_{2}, \\
a_{1}<2 b_{1} & \text { либо } & a_{3}<2 b_{3} ;
\end{array}
$$

$\Omega_{V}^{\prime \prime}-$ множество матриц из $\Omega_{V}$, у которых

$$
b_{2} \leqslant \frac{a_{2}}{2}+c_{2}, \quad c_{3} \leqslant \frac{a_{3}}{2}+b_{3}
$$

$\Omega_{V, 3}-$ множество матриц из $\Omega_{V}$, у которых

$$
a_{1}<\frac{b_{1}}{2}+c_{1}, \quad a_{2}+\frac{c_{2}}{2}<b_{2}, \quad 2 a_{3}<b_{3}+c_{3} .
$$

Сразу отметим, что все эти множества удовлетворяют условиям (А), (Б) из $§ 1$.

Пусть

$$
E_{\mathfrak{M}}(N)=\frac{1}{\# \mathscr{L}_{3}(\mathbb{Z} ; N)} \sum_{\Gamma \in \mathscr{L}_{3}(\mathbb{Z} ; N)} \# \mathfrak{M}(\Gamma)
$$

- среднее количество относительных минимумов решеток из $\mathscr{L}_{3}(\mathbb{Z} ; N)$. Из [15], [17] (см. также [16]) вытекает следующая асимптотическая формула:

$$
E_{\mathfrak{M}}(N)=\frac{4 \cdot C_{\mathfrak{M}}}{\zeta(2) \zeta(3)} \cdot \ln ^{2} N+O(\chi(N) \cdot \ln N+1),
$$

где

$$
C_{\mathfrak{M}}=\mu\left(\Omega_{1}\right)+\mu\left(\Omega_{2}\right)+2 \mu\left(\Omega_{3}\right)+2 \mu\left(\Omega_{4}\right) .
$$

В этом параграфе мы докажем следующий результат.

ТеОрема 3.1. Для любого иелого $N>1$ справедлива следующая асимптотическая формула для среднего числа вершин многогранников Клейна:

$$
E_{\mathscr{V}}(N)=\frac{4 \cdot C_{\mathscr{V}}}{\zeta(2) \zeta(3)} \cdot \ln ^{2} N+O(\chi(N) \cdot \ln N),
$$

əдe

$$
C_{\mathscr{V}}=C_{\mathfrak{M}}-\mu\left(\Omega_{V}^{\prime}\right)-3 \cdot \mu\left(\Omega_{V}^{\prime \prime}\right)-2 \cdot \mu\left(\Omega_{V, 3}\right) .
$$

Теорема будет доказана в конце параграфа.

ЗАмечАниЕ 3.1. Множество $\Omega_{V}^{\prime}$ можно представить в виде

$$
\Omega_{V}^{\prime}=\Omega_{V} \backslash\left(\Omega_{V}^{\prime \prime} \cup \Omega_{V}^{\mathrm{I}} \cup \Omega_{V}^{\mathrm{II}}\right),
$$

где $\Omega_{V}^{\mathrm{I}}\left(\Omega_{V}^{\mathrm{II}}\right)$ - множество матриц из $\Omega_{V}$, у которых

$$
a_{1} \geqslant 2 c_{1}, \quad a_{2} \geqslant 2 c_{2} \quad\left(a_{1} \geqslant 2 b_{1}, \quad a_{3} \geqslant 2 b_{3}\right) .
$$


Множества $\mathscr{P}\left(\Omega_{V}^{\prime \prime}\right), \mathscr{P}\left(\Omega_{V}^{\mathrm{I}}\right), \mathscr{P}\left(\Omega_{V}^{\mathrm{II}}\right)$ попарно не пересекаются. Преобразование, которое сначала меняет местами 2-ю и 3 -ю строки матрицы, а потом 2-й и 3-й столбцы, осуществляет биекцию между $\Omega_{V}^{\mathrm{I}}$ и $\Omega_{V}^{\mathrm{II}}$. Поэтому

$$
\begin{gathered}
\mu\left(\Omega_{V}^{\prime}\right)=\mu\left(\Omega_{V}\right)-\mu\left(\Omega_{V}^{\prime \prime}\right)-2 \cdot \mu\left(\Omega_{V}^{\mathrm{I}}\right), \\
C_{\mathscr{V}}=C_{\mathfrak{M}}-\mu\left(\Omega_{V}\right)-2 \mu\left(\Omega_{V}^{\prime \prime}\right)-2 \mu\left(\Omega_{V, 3}\right)+2 \mu\left(\Omega_{V}^{\mathrm{I}}\right) .
\end{gathered}
$$

ЗАмечание 3.2 . По-видимому, постоянные $C_{\mathfrak{M}}, C_{\mathscr{V}}$ не выражаются через известные. Приближенные вычисления дали следующие результаты:

$$
\begin{aligned}
\mu\left(\Omega_{1}\right) & \approx 0.0443899, \quad \mu\left(\Omega_{2}\right) \approx 0.09221, \quad \mu\left(\Omega_{3}\right) \approx 0.11194, \quad \mu\left(\Omega_{4}\right) \approx 0.02688, \\
\mu\left(\Omega_{V}\right) & \approx 0.00248, \quad \mu\left(\Omega_{V}^{\prime \prime}\right) \approx 0.00049, \quad \mu\left(\Omega_{V, 3}\right) \approx 0.00017, \quad \mu\left(\Omega_{V}^{\mathrm{I}}\right) \approx 0.00022, \\
C_{\mathfrak{M}} \approx 0.41205, \quad C_{\mathscr{V}} \approx 0.40867 . &
\end{aligned}
$$

ЗАмЕЧАНИЕ 3.3. Есть основания полагать, что для любой размерности $s$ существует предел

$$
\theta(s)=1-\lim _{N \rightarrow+\infty} \frac{\sum_{\Gamma \in \mathscr{L}_{s}(\mathbb{Z} ; N)} \# \mathscr{V}(\Gamma)}{\sum_{\Gamma \in \mathscr{L}_{s}(\mathbb{Z} ; N)} \# \mathfrak{M}(\Gamma)}
$$

- доля относительных минимумов, которые не являются вершинами многогранников Клейна $s$-мерных решеток. Известно, что $\theta(2)=0$. Из приведенных результатов вытекает, что $\theta(3) \approx 0.0082$. Начиная с размерности $s=4$ почти ничего неизвестно. Интересными представляются следующие вопросы.

1) Верно ли, что функиия $\theta$ монотонно возрастает?

2) Верно ли, что $\theta(s) \rightarrow 1$ при $s \rightarrow+\infty$ ?

Положительные ответы на эти вопросы означают, что с ростом размерности конструкции "относительные минимумы" и "многогранники Клейна" начинают сколь угодно сильно отличаться.

\section{Доказательство основного результата. Пусть}

$$
\mathscr{U}_{+}(N)=\left\{(\Gamma, a): \Gamma \in \mathscr{L}_{3}(\mathbb{Z} ; N), a \in \mathfrak{M}(\Gamma) \backslash \mathscr{V}(\Gamma), a \in(0,+\infty)^{3}\right\} .
$$

Используя вложение $(0.1)$ и учитывая, что у любой решетки из $\mathscr{L}_{3}(\mathbb{Z} ; N)$ количество относительных минимумов, имеющих хотя бы одну нулевую координату, оценивается величиной $O(\ln N+1)$ (см., например, [12], [20]), получаем

$$
E_{\mathscr{V}}(N)=E_{\mathfrak{M}}(N)-2^{3} \cdot \frac{\# \mathscr{U}_{+}(N)}{\# \mathscr{L}_{3}(\mathbb{Z} ; N)}+O(\ln N) .
$$

Здесь и ниже считаем, что $N \in \mathbb{N}, N>1$. Осталось вычислить количество минимумов, которые не являются вершинами многогранников Клейна.

Пусть $U(N)$ - множество пар $(\Gamma, a)$, удовлетворяющих следующим условиям:

1) $\Gamma \in \mathscr{L}_{3}(\mathbb{Z} ; N), a \in \Gamma \cap(0,+\infty)^{3}$;

2) существует такая треугольная грань $F=F(\Gamma, a)$ многогранника Клейна решетки $\Gamma$, что для некоторого $n \in \mathbb{N}$

$$
\begin{gathered}
u=(2 n+1) a-n b^{\prime}-n c^{\prime}, \\
F \cap \Gamma=\left\{b^{\prime}, c^{\prime}, u\right\} \cup\left\{\frac{k a+(n-k) u}{n}\right\}_{k=1}^{n},
\end{gathered}
$$

где $b^{\prime}, c^{\prime}, u$ - вершины $F$. 
Условие (3.3) означает, что узел а лежит на медиане, опущенной из вершины $u$ треугольника $F$ на сторону $b^{\prime} c^{\prime}$, условие $(3.4)-$ узлы из $\Gamma \cap F$, не являющиеся вершинами $F$, содержатся в отрезке $[a, u]$.

Докажем, что множества $\mathscr{U}_{+}(N)$ и $U(N)$ "почти" совпадают.

TeOpema 3.2. Ecлu $(\Gamma, a) \in U(N), m o(\Gamma, a) \in \mathscr{U}_{+}(N)$.

Если $(\Gamma, a) \in \mathscr{U}_{+}(N)$, причем множество вершин параллелепипеда

$$
\left[0,2 a_{1}\right] \times\left[0,2 a_{2}\right] \times\left[0,2 a_{3}\right]
$$

не содержит точек решетки $\Gamma$, кроме 0 и $2 a$, то $(\Gamma, a) \in U(N)$.

Доказательство теоремы вытекает из результатов [18].

Лемма 3.1. Пусть $\Gamma \in \mathscr{L}_{s}(\mathbb{Z} ; N), P_{i}, p_{i} \in(0,+\infty), p_{i} \leqslant P_{i}, i=\overline{1, s-1}$. Тогда количество минимумов $\gamma \in \mathfrak{M}(\Gamma)$, удовлетворяющих условиям

$$
p_{i} \leqslant\left|\gamma_{i}\right| \leqslant P_{i}, \quad i=\overline{1, s-1}
$$

не больие чем

$$
O_{s}\left(\prod_{i=1}^{s-1} \ln \left(\frac{P_{i}}{p_{i}}+1\right)\right) .
$$

Лемма легко доказывается методами [12] (см., например, [20]).

Из леммы 3.1 и известной оценки

$$
\left|\gamma_{i}\right| \leqslant N, \quad i=\overline{1, s} \quad \text { при } \quad \gamma \in \mathfrak{M}(\Gamma), \quad \Gamma \in \mathscr{L}_{s}(\mathbb{Z} ; N)
$$

вытекает следующее утверждение.

СлеДСтвиЕ 3.1. Для любой решетки $\Gamma \in \mathscr{L}_{s}(\mathbb{Z} ; N)$ количество относительных минимумов, у которых хотя бы одна координата равна некоторому фиксированному числу, не больше чем $O_{s}\left(\ln ^{s-2} N\right)$.

Лемма 3.2. Пусть $\Gamma \in \mathscr{L}_{3}(\mathbb{Z} ; N), \mathfrak{M}^{\prime}(\Gamma)$ - множество $а \in \mathfrak{M}(\Gamma) \cap[0,+\infty)^{3}$ таких, что хотя бы одна вершина параллелепипеда (3.5), отличная от 0 и $2 a$, является узлом Г. Тогда \# $\mathfrak{M}^{\prime}(\Gamma) \ll \ln N$.

ДокАЗАТЕЛЬСтво. Пусть $a \in \mathfrak{M}^{\prime}(\Gamma)$. Тогда одна из точек

$$
\left(2 a_{1}, 0,0\right), \quad\left(0,2 a_{2}, 0\right), \quad\left(0,0,2 a_{3}\right)
$$

является узлом Г. Пусть, например, $\left(2 a_{1}, 0,0\right) \in \Gamma$. Тогда $\left(2 a_{1}, 0,0\right) \in \mathfrak{M}(\Gamma)$, так как в противном случае существует узел $\gamma=\left(\gamma_{1}, 0,0\right) \in \Gamma$ такой, что $0<\gamma_{1}<2 a_{1}$ и точка $(a-\gamma)$ нарушает минимальность $a$. Минимум вида $\left(2 a_{1}, 0,0\right)$ единственен. Поэтому одна из координат $а$ однозначно определяется решеткой Г. Используя следствие 3.1 , получаем утверждение леммы.

Из леммы 3.2 и теоремы 3.2 вытекает оценка

$$
\# \mathscr{U}_{+}(N)=\# U(N)+O\left(\# \mathscr{L}_{3}(\mathbb{Z} ; N) \cdot \ln N\right) .
$$


Подставляя ее в (3.2), заключаем

$$
E_{\mathscr{V}}(N)=E_{\mathfrak{M}}(N)-8 \cdot \frac{\# U(N)}{\# \mathscr{L}_{3}(\mathbb{Z} ; N)}+O(\ln N) .
$$

Осталось вычислить количество элементов множества $U(N)$.

Лемма 3.3. Пусть $(\Gamma, a) \in U(N),\left(b^{\prime}, c^{\prime}, u\right)$ - вериины грани $F=F(\Gamma, a)$, удовлетворяющие (3.3), (3.4). Тогда

$$
3 a_{i} \geqslant b_{i}^{\prime}+c_{i}^{\prime}, \quad i=1,2,3,
$$

и существует перестановка $(k, m, n)$ из $\{1,2,3\}$ такая, что

$$
\begin{gathered}
b_{k}^{\prime}+c_{k}^{\prime}<a_{k}, \quad c_{m}^{\prime}<a_{m}<b_{m}^{\prime}, \quad b_{n}^{\prime}<a_{n}<c_{n}^{\prime}, \\
2 a_{m}<b_{m}^{\prime}, \quad 2 a_{n}<c_{n}^{\prime} .
\end{gathered}
$$

ДоказАтельство. Из (3.3) вытекает, что $\left(3 a-b^{\prime}-c^{\prime}\right) \in[0,+\infty)^{3}$. Поэтому выполняется (3.7). Кроме того,

$$
\left(b^{\prime}+c^{\prime}-a\right),\left(2 a-b^{\prime}\right),\left(2 a-c^{\prime}\right) \notin[0,+\infty)^{3} .
$$

Из первого условия следует, что найдется номер $k$ такой, что выполняется первое из неравенств (3.8). Пусть $\{m, n\}=\{1,2,3\} \backslash\{k\}$. Если $a_{m} \geqslant \max \left\{b_{m}^{\prime}, c_{m}^{\prime}\right\}$, то (в силу 2-го и 3-го соотношений из (3.10)) $2 a_{k}<\min \left\{b_{k}^{\prime}, c_{k}^{\prime}\right\}$. Противоречие с (3.7). Поэтому

$$
a_{m}<\max \left\{b_{m}^{\prime}, c_{m}^{\prime}\right\}, \quad a_{n}<\max \left\{b_{n}^{\prime}, c_{n}^{\prime}\right\} .
$$

Если $a_{m}<b_{m}^{\prime}, a_{n}<b_{n}^{\prime}$ (либо $a_{m}<c_{m}^{\prime}, c_{n}^{\prime}<b_{n}^{\prime}$ ), то из (3.7) вытекает, что $\left(2 a-c^{\prime}\right) \in[0,+\infty)^{3}$ (либо $\left.\left(2 a-b^{\prime}\right) \in[0,+\infty)^{3}\right)$. Противоречие. Значит, существуют номера $k, m, n$, удовлетворяющие (3.8). Неравенства (3.9) следуют из (3.8), (3.10). Лемма доказана.

Пусть $U_{1}(N)$ - множество пар $(\Gamma, a) \in U(N)$ таких, что грань $F=F(\Gamma, a)$ удовлетворяет условию $d(\Gamma ; F)=1$. Напомним, что $d(\Gamma ; F)$ совпадает с индексом подрешетки, порожденной векторами $a, b^{\prime}, c^{\prime}\left(b^{\prime}, c^{\prime}\right.$ - вершины треугольника $F$, удовлетворяющие $(3.3),(3.4))$. Разобьем $U_{1}(N)$ на две непересекающиеся части $U^{\prime}(N)$ и $U^{\prime \prime}(N)$, где $U^{\prime}(N)$ состоит из таких пар, что в условиях $(3.3)$, (3.4) $n=1$ (т.е. грань $F$ из узлов решетки содержит только свои вершины и $a$ ), а для $U^{\prime \prime}(N)$, соответственно, $n \geqslant 2$.

Лемма 3.4. Справедлива формула

$$
\# U^{\prime}(N)=\# \Omega_{V}^{\prime}(\mathbb{Z} ; N),
$$

где $\Omega_{V}^{\prime}(\mathbb{Z} ; N)$ - множество целочисленных матрии, $M \in \Omega_{V}^{\prime}$ с $\operatorname{det} M=N$.

Доказательство. Возьмем любую пару $(\Gamma, a) \in U^{\prime}(N)$. Пусть $F=F(\Gamma ; a)$. Согласно лемме 3.3 мы всегда можем единственным образом занумеровать вершины $F$ через $b^{\prime}, c^{\prime}, u$ так, что будут выполнены неравенства

$$
\begin{gathered}
b_{1}^{\prime}+c_{1}^{\prime}<a_{1}, \quad c_{2}^{\prime}<a_{2}<b_{2}^{\prime}, \quad b_{3}^{\prime}<a_{3}<c_{3}^{\prime}, \\
2 a_{2}<b_{2}^{\prime}, \quad 2 a_{3}<c_{3}^{\prime} .
\end{gathered}
$$


Определим взаимно однозначное отображение $\Phi$, которое каждой паре $(\Gamma, a) \in U^{\prime}(N)$ ставит в соответствие матрицу $M=M\left(a, b^{\prime}-a, c^{\prime}-a\right)$, где $b^{\prime}, c^{\prime}$ определяются указанным выше способом. Осталось доказать, что

$$
\Phi\left(U^{\prime}(N)\right)=\Omega_{V}^{\prime}(\mathbb{Z} ; N)
$$

1. Докажем, что $\Phi\left(U^{\prime}(N)\right) \subset \Omega_{V}^{\prime}(\mathbb{Z} ; N)$. Пусть $(\Gamma, a) \in U^{\prime}(N), M=M\left(a, b^{\prime}-a\right.$, $\left.c^{\prime}-a\right)=\Phi(\Gamma, a)$. Тогда выполняются неравенства $(3.7),(3.12)$. Кроме того, так как узлы $\left(5 a-2 b^{\prime}-2 c^{\prime}\right),\left(2 b^{\prime}-a\right),\left(2 c^{\prime}-a\right)$ не принадлежат $[0,+\infty)^{3}$, то

$$
\begin{array}{rll}
5 a_{2}<2\left(b_{2}^{\prime}+c_{2}^{\prime}\right) & \text { либо } & 5 a_{3}<2\left(b_{3}^{\prime}+c_{3}^{\prime}\right), \\
a_{1}>2 b_{1}^{\prime} & \text { либо } & a_{3}>2 b_{3}^{\prime}, \\
a_{1}>2 c_{1}^{\prime} & \text { либо } & a_{2}>2 c_{2}^{\prime} .
\end{array}
$$

Из (3.7), (3.12), (3.14) (и условий $\left.a \in(0,+\infty)^{3}, b^{\prime}, c^{\prime} \in[0,+\infty)^{3}\right)$ следует, что $M \in \Omega_{V}^{\prime}$. Так как $d(\Gamma ; F)=1$, то $a, b^{\prime}, c^{\prime}-$ базис $\Gamma$. Поэтому $\operatorname{det} M=N$. Значит, $M \in \Omega_{V}^{\prime}(\mathbb{Z} ; N)$ и $\Phi\left(U^{\prime}(N)\right) \subset \Omega_{V}^{\prime}(\mathbb{Z} ; N)$.

2. Осталось доказать, что $\Omega_{V}^{\prime}(\mathbb{Z} ; N) \subset \Phi\left(U^{\prime}(N)\right)$. Пусть $M=M(a, b, c) \in$ $\Omega_{V}^{\prime}(\mathbb{Z} ; N)$. Положим $b^{\prime}=b+a, c^{\prime}=c+a$ и определим решетку $\Gamma \in \mathscr{L}_{3}(\mathbb{Z} ; N)$ с базисом $a, b, c$, а также узел $u=3 a-b^{\prime}-c^{\prime}$ и треугольник $F$ с вершинами в точках $b^{\prime}, c^{\prime}, u$. Тогда

$$
\Gamma \cap F=\left\{a, b^{\prime}, c^{\prime}, u\right\} .
$$

Кроме того, $a \in(0,+\infty)^{3}, b^{\prime}, c^{\prime} \in[0,+\infty)^{3}$ и выполняются неравенства (3.7), (3.12), (3.14). Применим лемму 2.1. Учитывая, что минимальная $X(\Gamma ; F)$-окрестность $F$ состоит из точек

$$
\left(2 c^{\prime}-a\right), \quad\left(2 b^{\prime}-a\right), \quad\left(2 a-b^{\prime}\right), \quad\left(2 a-c^{\prime}\right), \quad\left(5 a-2 b^{\prime}-2 c^{\prime}\right), \quad\left(b^{\prime}+c^{\prime}-a\right),
$$

получаем, что $F$ - грань многогранника Клейна решетки $\Gamma$. Поэтому $(\Gamma, a) \in$ $U^{\prime}(N)$ и, значит, $\Omega_{V}^{\prime}(\mathbb{Z} ; N) \subset \Phi\left(U^{\prime}(N)\right)$. Равенство (3.13) доказано. Лемма доказана.

Лемма 3.5. Справедлива формула

$$
\# U^{\prime \prime}(N)=3 \cdot \# \Omega_{V}^{\prime \prime}(\mathbb{Z} ; N),
$$

где $\Omega_{V}^{\prime \prime}(\mathbb{Z} ; N)$ - множество целочисленных матрии, $M \in \Omega_{V}^{\prime \prime} c \operatorname{det} M=N$.

ДокАзАтельство. Согласно лемме 3.3 множество $U^{\prime \prime}(N)$ можно разбить на три непересекающиеся части $U_{k}^{\prime \prime}(N), k=1,2,3$, так, что

$$
\forall(\Gamma, a) \in U_{k}^{\prime \prime}(N) \quad a_{k}>b_{k}^{\prime}+c_{k}^{\prime},
$$

где $b^{\prime}, c^{\prime}$ - вершины $F=F(\Gamma, a)$, удовлетворяющие $(3.3),(3.4)$. Очевидно, что

$$
\# U_{1}^{\prime \prime}(N)=\# U_{2}^{\prime \prime}(N)=\# U_{3}^{\prime \prime}(N), \quad \# U^{\prime \prime}(N)=3 \cdot \# U_{1}^{\prime \prime}(N) .
$$

Вычислим \# $U_{1}^{\prime \prime}(N)$. Согласно лемме 3.3 для любой пары $(\Gamma, a) \in U_{1}^{\prime \prime}(N)$ существует единственная нумерация вершин грани $F=F(\Gamma, a)$ через $b^{\prime}, c^{\prime}, u$ так, 
что выполняются (3.3), (3.4), (3.12). Определим взаимно однозначное отображение $\Phi$, которое каждой паре $(\Gamma, a) \in U_{1}^{\prime \prime}(N)$ ставит в соответствие матрицу $M=M\left(a, b^{\prime}-a, c^{\prime}-a\right)$, где $b^{\prime}, c^{\prime}$ определяются указанным выше способом. Осталось показать, что $\Phi$ отображает $U_{1}^{\prime \prime}(N)$ на $\Omega_{V}^{\prime \prime}(\mathbb{Z} ; N)$.

1. Пусть $(\Gamma, a) \in U^{\prime \prime}(N), M=M\left(a, b^{\prime}-a, c^{\prime}-a\right)=\Phi(\Gamma, a)$. Так как $(5 a-$ $\left.2 b^{\prime}-2 c^{\prime}\right) \in[0,+\infty)^{3}$, то помимо (3.7), (3.12) выполняются оценки

$$
5 a_{2} \geqslant 2\left(b_{2}^{\prime}+c_{2}^{\prime}\right), \quad 5 a_{3} \geqslant 2\left(b_{3}^{\prime}+c_{3}^{\prime}\right) .
$$

Используя (3.7), (3.12), (3.16), получаем $M \in \Omega_{V}^{\prime \prime}$. Так как $d(\Gamma ; F)=1$, то $a, b^{\prime}, c^{\prime}-$ базис $\Gamma$. Кроме того, $\operatorname{det} M>0$, и поэтому $\operatorname{det} M=N$. Значит, $M \in \Omega_{V}^{\prime \prime}(\mathbb{Z} ; N)$ и $\Phi\left(U^{\prime \prime}(N)\right) \subset \Omega_{V}^{\prime \prime}(\mathbb{Z} ; N)$.

2. Пусть $M(a, b, c) \in \Omega_{V}^{\prime \prime}(\mathbb{Z} ; N)$. Положим $b^{\prime}=b+a, c^{\prime}=c+a$ и определим решетку $\Gamma \in \mathscr{L}_{3}(\mathbb{Z} ; N)$ с базисом $a, b, c$ и треугольник $F$ с вершинами в точках $b^{\prime}, c^{\prime}, u$, где $u=(2 n+1) a-n\left(b^{\prime}+c^{\prime}\right), n$ - наибольшее натуральное число, для которого соответствующий $u$ имеет неотрицательные координаты. Тогда выполняются (3.4), (3.7), (3.12), (3.16). Минимальная $X(\Gamma ; F)$-окрестность $F$ состоит из четырех точек

$$
\left(2 a-b^{\prime}\right), \quad\left(2 a-c^{\prime}\right), \quad\left((2 n+3) a-(n+1)\left(b^{\prime}+c^{\prime}\right)\right), \quad\left(b^{\prime}+c^{\prime}-a\right) .
$$

Используя лемму 2.1, получаем, что $F$ - грань многогранника Клейна решетки $\Gamma$. Значит, $(\Gamma, a) \in U_{1}^{\prime \prime}(N)$, причем $M=\Phi(\Gamma, a)$. Следовательно, $\Omega_{V}^{\prime \prime}(\mathbb{Z} ; N) \subset \Phi\left(U_{1}^{\prime \prime}(N)\right)$. Поэтому $\Phi\left(U_{1}^{\prime \prime}(N)\right)=\Omega_{1}^{\prime \prime}(\mathbb{Z} ; N), \# U_{1}^{\prime \prime}(N)=\# \Omega_{V}^{\prime \prime}(\mathbb{Z} ; N)$. Применяя (3.15), приходим к требуемой формуле. Лемма доказана.

Осталось рассмотреть множество $U(N) \backslash U_{1}(N)$. В [11] получено полное описание типов граней трехмерных полиэдров Клейна с $d(\Gamma ; F)>1$. Согласно результатам [11], если $(\Gamma, a) \in U(N), F=F(\Gamma, a)$, причем $d(\Gamma ; F)>1$, то $d(\Gamma ; F)=3$ и грань $F$ принадлежит типу $\mathscr{T}_{3}$, где $\mathscr{T}_{3}$ - множество многоугольников целочисленно-линейно эквивалентных треугольнику с вершинами в точках

$$
(1,1,3), \quad(0,2,3), \quad(2,3,3) \text {. }
$$

Отметим, что тогда $a$ - единственная точка $\Gamma$, лежащая на $F(\Gamma, a)$ и не совпадающая с вершиной (т.е. $n=1$ ).

Лемма 3.6. Пусть $U_{3}(N)=U(N) \backslash U_{1}(N)$, а $\omega_{V, 3}(N)$ - множество иелочисленных матрии, $M=M(a, b, c) \in \Omega_{V, 3}$, удовлетворяющих условиям

$$
\operatorname{det} M=3 N, \quad(a-b+c) \in 3 \mathbb{Z}^{3} .
$$

Тогда $\# U_{3}(N)=2 \cdot \# \omega_{V, 3}(N)$.

ДокАзАтЕльство. Согласно лемме 3.3 для любой пары $(\Gamma ; a) \in U_{3}(N)$ существует единственная нумерация вершин треугольника $F=F(\Gamma ; a)$ через $b^{\prime}$, $c^{\prime}, u$ так, что выполняются (3.12). Так как $F$ имеет тип $\mathscr{T}_{3}$, то

$$
\frac{a-b^{\prime}+c^{\prime}}{3} \in \Gamma \quad \text { либо } \quad \frac{a+b^{\prime}-c^{\prime}}{3} \in \Gamma .
$$


Одновременно эти включения выполняться не могут (иначе $2 a / 3 \in \Gamma$ - противоречие с $a \in \mathfrak{M}(\Gamma))$. Поэтому $U_{3}(N)$ можно разбить на две непересекающиеся части $U_{3,1}(N)$ и $U_{3,2}(N)$ так, что для первого множества выполняется первое из включений (3.18), а для второго - второе. Очевидно, что

$$
\# U_{3,1}(N)=\# U_{3,2}(N), \quad \# U_{3}(N)=2 \cdot \# U_{3,1}(N) .
$$

Определим взаимно однозначное отображение $\Phi$, которое паре $(\Gamma, F) \in$ $U_{3,1}(N)$ ставит в соответствие матрицу $M\left(a, b^{\prime}-a, c^{\prime}-a\right)$, где $b^{\prime}, c^{\prime}$ определяются указанным выше образом. Осталось доказать, что $\Phi\left(U_{3,1}(N)\right)=\omega_{V, 3}(N)$.

1. Пусть $(\Gamma, a) \in U_{3,1}(N)$. Тогда выполняются (3.7), (3.12), (3.17). Узлы $\widetilde{a}$, $b, c$ образуют базис $\Gamma$, где

$$
\widetilde{a}=\frac{a-b+c}{3}, \quad b=b^{\prime}-a, \quad c=c^{\prime}-a .
$$

Кроме того,

$$
b_{1}^{\prime}+2 c_{1}^{\prime}<a_{1}, \quad 5 a_{2}<c_{2}^{\prime}+2 b_{2}^{\prime}, \quad 2 a_{3}+b_{3}^{\prime}<c_{3}^{\prime} .
$$

Для доказательства (3.21) достаточно рассмотреть узлы

$$
\begin{gathered}
u^{(1)}=2 \widetilde{a}+b=\frac{2}{3}\left(a+\frac{b}{2}+c\right)=\frac{-a+b^{\prime}+2 c^{\prime}}{3}, \\
u^{(2)}=2 \widetilde{a}-c=\frac{2}{3}\left(a-b-\frac{c}{2}\right)=\frac{5 a-2 b^{\prime}-c^{\prime}}{3}, \\
u^{(3)}=2 \widetilde{a}+b-c=\frac{2}{3}\left(a+\frac{b}{2}-\frac{c}{2}\right)=\frac{2 a+b^{\prime}-c^{\prime}}{3},
\end{gathered}
$$

которые не должны принадлежать $[0,+\infty)^{3}$. Используя указанные соотношения, получаем $M=M\left(a, b^{\prime}-a, c^{\prime}-a\right) \in \omega_{V, 3}(N)$. Значит, $\Phi\left(U_{3,1}(N)\right) \subset \omega_{V, 3}(N)$.

2. Пусть $M(a, b, c) \in \omega_{V, 3}(N)$. Определим $b^{\prime}=b+a, c^{\prime}=c+a$ и решетку $\Gamma \in \mathscr{L}_{3}(\mathbb{Z} ; N)$ с базисом $\widetilde{a}, b, c$, где $\widetilde{a}$ удовлетворяет (3.20). Тогда выполняются соотношения (3.7), (3.12), (3.17), (3.21). Пусть $F$ - треугольник с вершинами в точках $b^{\prime}, c^{\prime}, u$, где $u=3 a-\left(b^{\prime}+c^{\prime}\right)$. Тогда $F \subset[0,+\infty)^{3}$. Докажем, что $F$ - грань многогранника Клейна решетки Г. Будем использовать лемму 2.1. Множество $X(\Gamma ; F)$ состоит из точек вида

$$
3 \widetilde{a}+\frac{3 n}{2} b+\frac{3 m}{2} c=a+(b-a)\left(\frac{3 n}{2}-1\right)+(c-a)\left(\frac{3 m}{2}+1\right), \quad n, m \in \mathbb{Z},
$$

а также узлов $\Gamma$, лежащих на плоскости, проходящей через $F$. Поэтому минимальная $X(\Gamma ; F)$-окрестность $F$ состоит из точек $3 / 2 u^{(i)}, i=1,2,3$. Согласно (3.21) эти точки не лежат в $[0,+\infty)^{3}$. Используя лемму 2.1 , получаем, что $F$ - грань многогранника Клейна решетки $\Gamma$. Значит, $(\Gamma, a) \in U_{3,1}(N)$, причем $\Phi(\Gamma ; a)=M(a, b, c)$. Поэтому $\omega_{V, 3}(N) \subset \Phi\left(U_{3,1}(N)\right)$ и, следовательно, $\Phi\left(U_{3,1}(N)\right)=\omega_{V, 3}(N)$. Используя (3.19), получаем утверждение леммы. 
ДокАЗАТЕЛЬСТво тЕОРЕмы 3.2. Согласно леммам 3.4-3.6

$$
\# U(N)=\# \Omega_{V}^{\prime}(\mathbb{Z} ; N)+3 \cdot \# \Omega_{V}^{\prime \prime}(\mathbb{Z} ; N)+2 \cdot \# \omega_{V, 3}(N) .
$$

Применяя теорему 1.1 и следствие 1.1 , получаем

$$
\# U(N)=\frac{\left.\# \mathscr{L}_{3}(\mathbb{Z} ; N)\right)}{2 \zeta(2) \zeta(3)}\left(\left(\mu\left(\Omega_{V}^{\prime}\right)+3 \cdot \mu\left(\Omega_{V}^{\prime \prime}\right)+2 \cdot \mu\left(\Omega_{V, 3}\right)\right) \ln ^{2} N+O(\chi(N) \ln N)\right) .
$$

Из последнего равенства и (3.6), (3.1) вытекает утверждение теоремы.

\section{Список литературы}

[1] Г. Ф. Вороной, Собрание сочинений в 3-x томах. Т. 1, Изд-во АН УССР, Киев, 1952.

[2] H. Minkowski, "Généralisation de la théorie des fractions continues", Ann. Sci. École Norm. Sup. (3), 13:2 (1896), 41-60.

[3] F. Klein, "Ueber eine geometrische Auffassung der gewohnlichen Kettenbruchentwickelung", Gött. Nachr., 3 (1895), 357-359.

[4] В.А. Быковский, "Относительные минимумы решеток и вершины многогранников Клейна", Функи. анализ и его прил., 40:1 (2006), 69-71; англ. пер.: V.A. Bykovskii, "Local minima of lattices and vertices of Klein polyhedra", Funct. Anal. Appl., 40:1 (2006), 56-57.

[5] V.I. Arnold, "Higher dimensional continued fractions", Regul. Chaotic Dyn., 3:3 (1998), 10-17.

[6] В. И. Арнольд, Задачи Арнолъда, Фазис, М., 2000; англ. пер.: V. Arnold, Arnold's problems, Springer-Verlag, Berlin, 2004.

[7] В. И. Арнольд, Цепные дроби, МЦНМО, М., 2001.

[8] M. L. Kontsevich, Yu. M. Suhov, "Statistics of Klein polyhedra and multidimensional continued fractions", Pseudoperiodic topology, Amer. Math. Soc. Transl. Ser. 2, 197, Amer. Math. Soc., Providence, RI, 1999, 9-27.

[9] J.-O. Moussafir, Voiles et polyèdres de Klein: Géométrie, algorithmes et statistiques, Doc. Sci. Thése, Univ. Paris IX-Dauphine, Paris, 2000.

[10] О.Н. Карпенков, "Об инвариантной мере Мёбиуса и распределении граней Гаусса-Кузьмина", Анализ и особенности. Часть 1, Сборник статей. К 70-летию со дня рождения академика Владимира Игоревича Арнольда, Тр. МИАН, 258, Наука, M., 2007, 79-92; англ. пер.: О. N. Karpenkov, "On an invariant Mobius measure and the Gauss-Kuzmin face distribution", Proc. Steklov Inst. Math., 258 (2007), 74-86.

[11] O. N. Karpenkov, "Completely empty pyramids on integer lattices and two-dimensional faces of multidimensional continued fractions", Monatsh. Math., 152:3 (2007), 217-249.

[12] В. А. Быковский, "О погрешности теоретико-числовых квадратурных формул", Докл. РАН, 389:2 (2003), 154-155; англ. пер.: V. A. Bykovskii, "On the error of number-theoretic quadrature formulas", Dokl. Math., 67:2 (2003), 175-176.

[13] В. А. Быковский, "Отклонение сеток Коробова", Изв. РАН. Сер. матем., 76:3 (2012), 19-38; англ. пер.: V. A. Bykovskii, "The discrepancy of the Korobov lattice points", Izv. Math., 76:3 (2012), 446-465.

[14] А. А. Илларионов, Д. А. Слинкин, "О количестве вершин многогранников Клейна в среднем", Дальневост. матем. журн., 11:1 (2011), 48-55. 
[15] А.А. Илларионов, "Среднее количество относительных минимумов трехмерных целочисленных решеток”, Алгебра и анализ, 23:3 (2011), 189-215; англ. пер.: A. A. Illarionov, "Average number of local minima for three-dimensional integral lattices", St. Petersburg Math. J., 23:3 (2012), 551-570.

[16] А.А. Илларионов, "Среднее количество относительных минимумов трехмерных целочисленных решеток фиксированного определителя”, Изв. РАН. Сер. матем., 76:3 (2012), 111-138; англ. пер.: А. A. Illarionov, "The average number of relative minima of three-dimensional integer lattices of a given determinant", Izv. Math., 76:3 (2012), 535-562.

[17] A. A. Illarionov, "On the asymptotic distribution of integer matrices", Mosc. J. Comb. Number Theory, 1:4 (2011), 13-57.

[18] О.Н. Герман, "Полиэдры Клейна и относительные минимумы решеток", Матем. заметки, 79:4 (2006), 546-552; англ. пер.: O. N. German, "Klein polyhedra and relative minima of lattices", Math. Notes, 79:4 (2006), 505-510.

[19] А. А. Илларионов, "Статистические свойства многомерных аналогов непрерывных дробей”, Наука - Хабаровскому краю: материалы ХІІ краевого конкурса молодых ученых, Изд-во Тихоокеан. гос. ун-та, Хабаровск, 2010, 5-15.

[20] А. А. Илларионов, "Оценки количества относительных минимумов решеток", $M a$ тем. заметки., 89:2 (2011), 249-259; англ. пер.: А. A. Illarionov, "Estimates of the number of relative minima of lattices", Math. Notes, 89:2 (2011), 245-254.

\section{А. А. Илларионов (А. А. Illarionov)}

Хабаровское отделение

Института прикладной математики ДВО РАН

E-mail: illar_a@list.ru
Поступила в редакцию 01.12 .2010 и 18.03 .2013 